\title{
Sub arcsec evolution of solar magnetic fields
}

\author{
Th. Roudier ${ }^{1}$, J. M. Malherbe ${ }^{2}$, J. Moity ${ }^{2}$, S. Rondi ${ }^{1}$, P. Mein ${ }^{2}$, and Ch. Coutard ${ }^{2}$ \\ 1 Laboratoire d'Astrophysique de l'Observatoire Midi-Pyrénées, Université Paul Sabatier Toulouse III, CNRS, 57 avenue d'Azeirex, \\ BP 826, 65008 Tarbes Cedex, France \\ 2 LESIA, Observatoire de Paris, Section de Meudon, 92195 Meudon, France
}

Received 3 February 2006 / Accepted 20 April 2006

\begin{abstract}
Context. The evolution of the concentrated magnetic field in flux tubes is one challenge of the nowadays Solar physics which requires time sequence with high spatial resolution.

Aims. Our objective is to follow the properties of the magnetic concentrations during their life, in intensity (continuum and line core), magnetic field and Doppler velocity.

Methods. We have observed solar region NOAA 0644 on 2004 July 15 at Pic du Midi observatory with the Multichannel Subtractive Double Pass (MSDP) operating mode and analysed the circular polarization of the Na D1 $589.6 \mathrm{~nm}$ spectral line in terms of longitudinal magnetic field in 2D field of view $\left(5.6^{\prime \prime} \times 80^{\prime \prime}\right)$, during $41 \mathrm{mn}$ at two altitudes $327 \mathrm{~km}$ and $170 \mathrm{~km}$ in the photosphere.

Results. Our data analysis reveals that all the concentrated magnetic features are associated with downward motions and the magnetic field remains very stable during the $41 \mathrm{mn}$. At different height 327 and $170 \mathrm{~km}$, a clear depth effect is visible on Doppler velocity but not in the magnetic field strength.
\end{abstract}

Key words. Sun: photosphere - Sun: granulation - Sun: magnetic fields

\section{Introduction}

The quiet-Sun magnetism which is present in the form of discrete element over the Sun surface plays an important role in the solar magnetic activity. The simulations (Sanchez Almeida et al. 2003) point out that more than $90 \%$ of the unsigned magnetic flux existing in their numerical data would not appear in magnetograms with $1^{\prime \prime}$ angular resolution. Thus, the existing flux in the quiet sun probably exceeds by far that carried by active regions during the maximum of the solar cycle (Sanchez Almeida et al. 2003). Then, one of the main goals of the solar physics today is to describe and understand the physical and dynamical properties of the magnetic flux tubes. These features which are elementary bricks of the magnetic field in the quiet-Sun (diameter around 100-150 km or less) require for their analysis very high spatial and temporal resolution observations in spectropolarimetric mode. Recent work (Rimmele 2004; Berger et al. 2004; Rouppe et al. 2005) demonstrated the gain of the large solar telescopes, $1 \mathrm{~m}$ class, to learn more about the magnetic flux tubes properties. With the high spatial resolution $\left(0 !^{\prime} 1\right)$ of their observations, they show that weak upflows are found in the flux sheets and downflows in the immediate surroundings and the visible appearance of the small magnetic features is determined by their horizontal size (micro-pores, ribbon shaped and flowers).

The purpose of our paper is to show the possibilities of the combination of a Nematic Liquid Crystal (NLC) polarimeter with MSDP spectrograph to study the temporal evolution of magnetic concentration with a spatial resolution of 0.3 to 0.4 . We organized the paper in two parts: the first part presents the statistical properties of the magnetic field relatively to the Doppler velocity and intensity distribution over the solar surface. We focus in particular on noise levels due to the reduction process. In the second part, we consider the time evolution properties of different magnetic features, such as magnetic concentrations and moving magnetic features (MMF).

\section{Observations}

We observed the active region NOAA $0644 \mu=0.91$ (East $24^{\circ}$, North $12^{\circ}$ ) close to the disc center, on 15st July 2004 between $6 \mathrm{~h} 34 \mathrm{mn} 46 \mathrm{~s}$ and $7 \mathrm{~h} 15 \mathrm{mn} 21 \mathrm{~s}$ UT. The duration of the analyzed sequence is $41 \mathrm{mn}$ with a mean time step of $60 \mathrm{~s}$. The exposure time was $40 \mathrm{~ms}$ with Camera LaVision CCD $(1280 \times 1024$ pixels $)$. The observation was performed in the NaD1 line $(\lambda 5896 \AA)$ with the Multichannel Subtractive Double Pass (MSDP) system (designed by P. Mein) operating on the solar spectrograph (designed by Z. Mouradian) at the focus of the $50 \mathrm{~cm}$ refractor of the Pic du Midi Observatory (Lunette Jean Rosch) as described by Mein (1981) and Mein (2002). The pixel size on the CCD camera is $0{ }^{\prime} 13$ and $0{ }^{\prime} .1$ on reduced data. The spectral resolution is $144 \mathrm{~m} \AA$ and 11 channels were recorded simultaneously. The elementary field of view is $90^{\prime \prime} \times 14^{\prime \prime}$, and $80^{\prime \prime} \times 5.6^{\prime \prime}$ after data reduction of the whole temporal sequence due to shifts in the pointing of the refractor. Polarization experiment setup is based on the one described by Malherbe et al. (2004), except that the ferroelectric crystal was replaced by a new nematic crystal.

Figures 1 and 2 show the general context of our field of view relatively to Meudon spectroheliogram and MDI/SOHO observations.

\section{Data reduction}

Data analysis was performed using the standard MSDP software developed by P. Mein and available on line at the BASS2000 data 


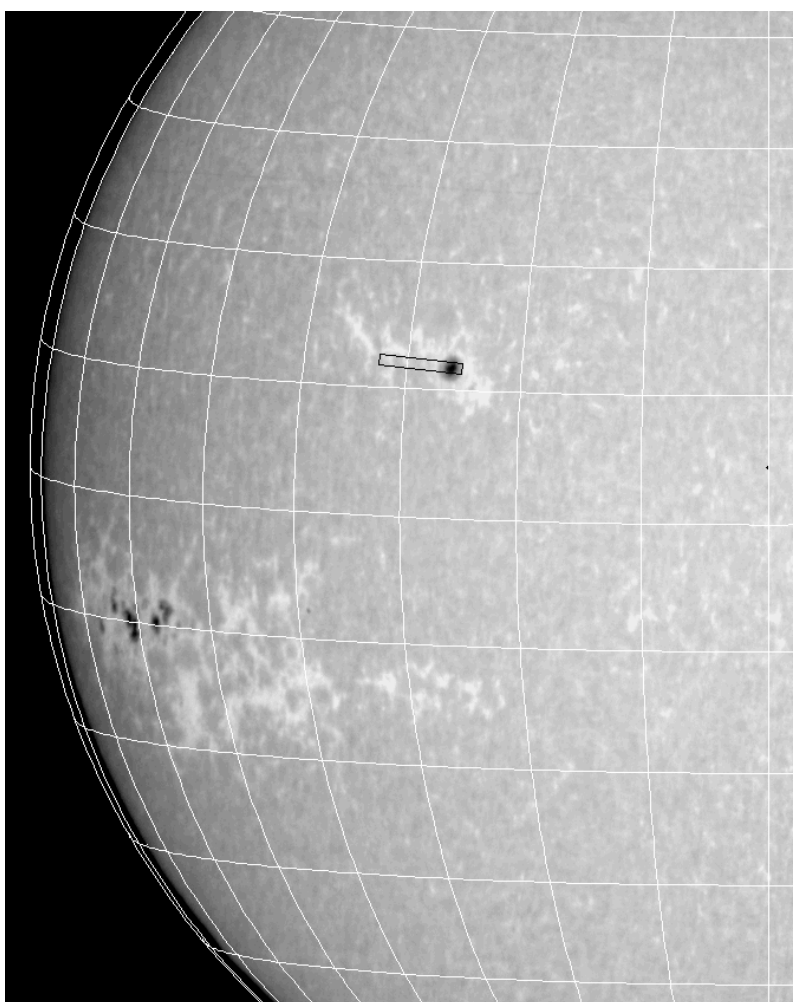

Fig. 1. Meudon spectroheliogram in CaII K1v (blue wing of CaII K $393.4 \mathrm{~nm}$ ), on 2004 July 14 at 07:36 UT, showing NOAA 0644. The black box corresponds to the MSDP field of view.

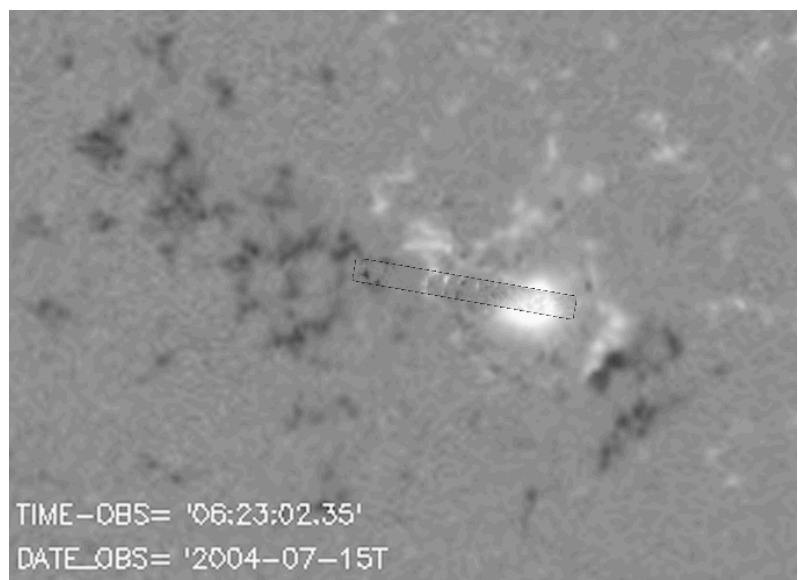

Fig. 2. SOHO/MDI magnetogram on 2004 July 15 at 06:23 UT, showing NOAA 0644. We observed with the MSDP the eastern part of the spot (the black box corresponds to the MSDP field of view); the magnetic field found by MDI in this spot was between -1000 and 1000 Gauss, in agreement with our results (courtesy SOHO/MDI).

base (see http://bass2000.bagn.obs-mip.fr). The different steps to analyse for each state of polarization have been described in a previous paper (Malherbe et al. 2004). The images were corrected for dark current and gain, rigidly aligned, destretched and filtered for p-modes in $k-\omega$ space (threshold phase velocity $=4 \mathrm{~km} \mathrm{~s}^{-1}$ ) to remove five-minute oscillations.

We determine the wavelength shift of each profile $I+V(\lambda)$ and $I-V(\lambda)$ using the classical bisector technique: the wavelength shift is the shift of the middle of a chord having a full width of $2 \times \delta \lambda(2 \times 144 \mathrm{~m} \AA$ for the chromosphere or $2 \times 288 \mathrm{~m} \AA$ for the photosphere). If we call $\lambda_{1}$ the shift of $I+V$ and $\lambda_{2}$ the shift of $I-V$, the dopplershift (i.e. the radial velocity $V_{/ /}$, (Fig. 4)) is given by $\Delta \lambda=\left(\lambda_{1}+\lambda_{2}\right) / 2$.

On the contrary, the Zeeman shift is proportional to $\Delta \lambda_{B}=$ $\left(\lambda_{1}-\lambda_{2}\right) / 2$. We calibrate the values of the magnetic field using the classical weak field approximation, i.e.

$\Delta \lambda_{B}=4.67 \times 10^{-13} B_{/ /} \lambda^{2} g$

where $B_{/ /}$is the magnetic field in Gauss, $\Delta \lambda_{B}$ the Zeeman shift in $\AA, \lambda$ the wavelength of the line in $\AA$ and $g$ the effective landé factor of the line (1.33).

For the Sodium lines, we obtain approximately the following correspondance between velocities and magnetic fields: $1 \mathrm{~m} / \mathrm{s}=$ 0.91 Gauss.

According to Roudier et al. (2001), the two computed positions chords of half width 144 and $288 \mathrm{~m} \AA$ in the spectral line correspond to the height 327 and $170 \mathrm{~km}$, values which were derived from modelling the radiative transfer of $\mathrm{NaD} 1$ through the VAL-C atmospheric stratification with no magnetic field.

The observing mode consists in bursts of $2 \times 30$ images $I+V$ and $I-V$ with very short exposure time $(40 \mathrm{~ms})$ in order to reduce seeing effects. Hence, the signal to noise ratio of individual images is small (about 100). It can be increased after image selection, destretching and summing (Fig. 3).

Several calculations were done on a unique burst of $2 \times 30 \mathrm{im}$ ages from which it was possible to select up to $2 \times 20$ fairly good images (in general it was never possible to extract more than $2 \times 5$ to $2 \times 10$ images). Before summing images, we corrected seeing induced translations and deformations using a destretching method (November 1986).

In order to estimate the noise on the magnetic field, we select a small area of quiet sun, with no visible magnetic structure, of $6^{\prime \prime} \times 6^{\prime \prime}$ (3600 pixels). In this area, the average value of the magnetic field is almost zero, and we take the standard deviation as an estimator of the noise.

We find the following results:

- at $144 \mathrm{~m} \AA$, for a single determination from one couple $I+V$, $I-V$, the noise is 60 Gauss. When we use 20 successive determinations (within a few seconds), after destretching and summing, it reduces to 20 Gauss.

- at $288 \mathrm{~m} \AA$, for a single determination from one couple $I+V$, $I-V$, the noise is 85 Gauss. When we use 20 successive determinations (within a few seconds), after destretching and summing, it reduces to 25 Gauss.

Obviously, when we use 20 determinations, we do not reduce the noise by a factor $\operatorname{sqrt}(20)=4.5$, but by a factor 3 only. The reason is that the destretching procedure is not fully efficient and has an impact on the spatial resolution of magnetic fields.

\section{Results}

Before describing in some detail the evolution of magnetic features, we first present the statistical properties of velocities and magnetic field of our field of view.

\subsection{Properties of quiet and bright feature regions (excluding spot)}

Using the magnetic field as a selection criterion, we selected magnetic flux concentrations greater than 60 Gauss, outside the sunspot (see example of Fig. 8), in order to perform a statistical analysis of the bright features region. 


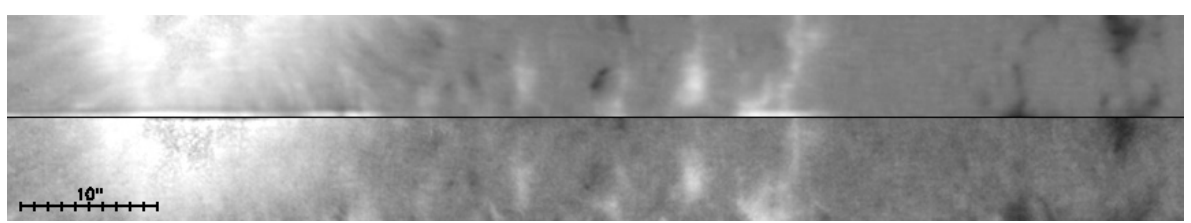

Fig. 3. Effect of image accumulation on the determination of magnetic fields (top: after summation of 20 determinations after destretching single; bottom: single determination). The field of view is $15^{\prime \prime} \times 86^{\prime \prime}$. A single determination requires a couple of images $I+V$ and $I-V$.

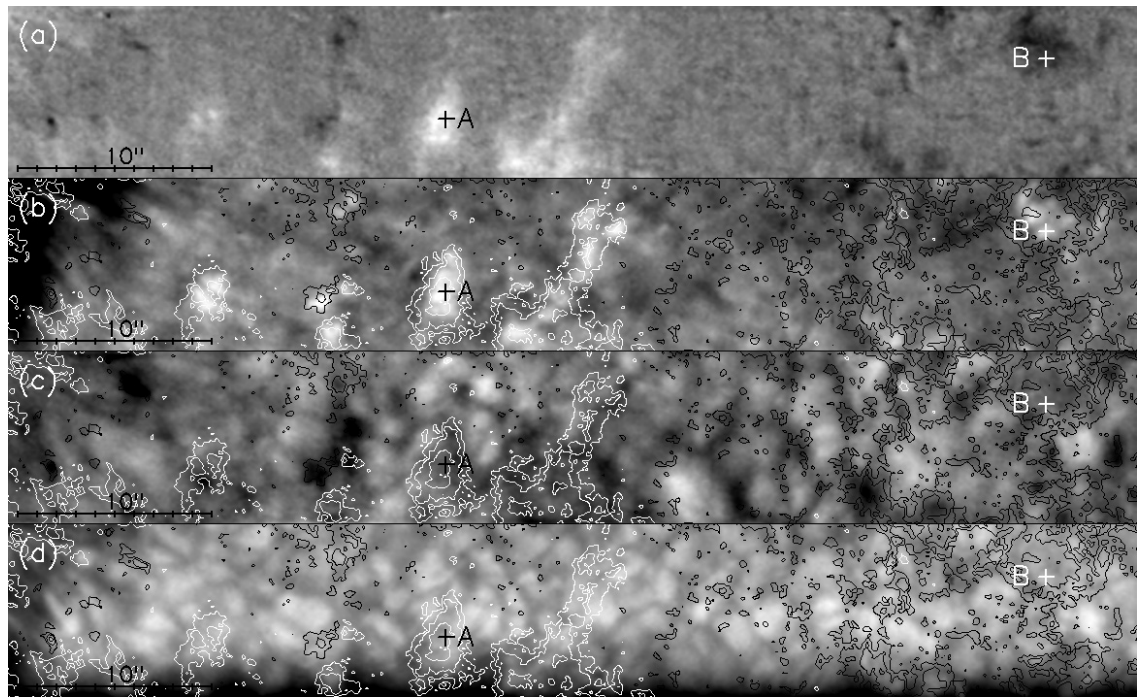

Fig. 4. Quiet area $58^{\prime \prime} \times 9^{\prime \prime}$ at $t=31 \mathrm{mn}$. From top to bottom: a) magnetic field $B_{/ /}$at $144 \mathrm{~m} \AA$ (high photosphere). North polarities in white, South in dark; b) intensity fluctuations (Filigree, bright features) at $144 \mathrm{~m} \AA$ (high photosphere); c) line-of-sight velocity $V_{/ /}$at $144 \mathrm{~m} \AA$ upflows are represented in white and downflows in dark (high photosphere); d) continuum intensity of NaD1 (first channel of the MSDP with a small variation in wavelength giving a darker part in the bottom of that figure) (photosphere). Isocontours of $B_{/ /}$at $144 \mathrm{~m} \AA$ are plotted for $\pm 150, \pm 300$ and \pm 600 Gauss. Magnetic concentrations are strongly correlated to bright features and filigree, and also to intergranular lanes (see right of the figure).

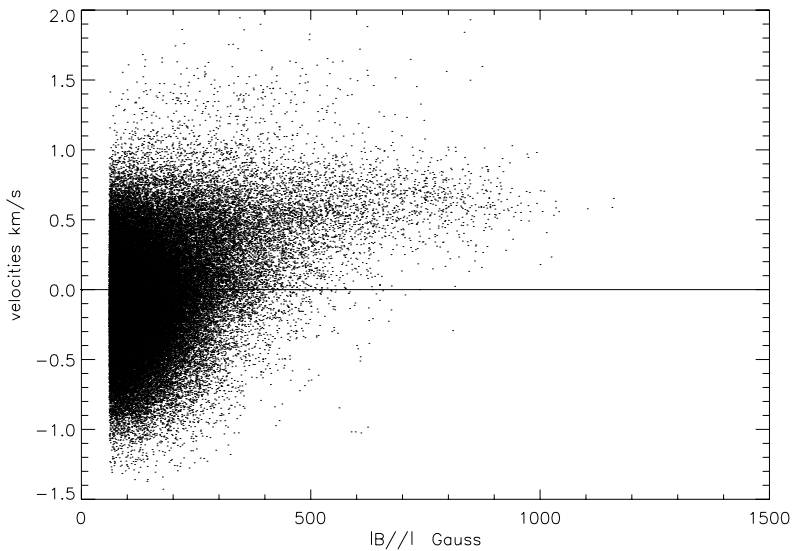

Fig. 5. Velocities versus magnetic field (absolute value) at $288 \mathrm{~m} \AA$ (170 km), $5 \mathrm{mn}$ filtered for $\left|B_{/ /}\right|>60$ Gauss. Positive velocity denotes downflow.

Figures 5-7 can be compared directly to Figs. 6 and 7 of Rimmele (2004) (scatter plot of $B_{/ /}$vs. velocity and intensity for magnetic flux concentration). For these figures positive velocity denotes downflow.

In Fig. 5, like Rimmele (2004), we observe an increase of magnetic field amplitude in the downflow. As $\left|B_{/ /}\right|$increases, the velocities remain stable around $0.7 \mathrm{~km} \mathrm{~s}^{-1}$ and do not turn to zero like observed by Rimmele (2004). The difference can be attributed to the different spatial resolution of the observations and the angle of viewing which contributes to mix the flux tube properties with those of its surrounding. In Fig. 6, like Rimmele (2004), we find a linear increase of bright feature intensity with the magnetic field. Figure 7 shows scatter plot of velocity vs. intensity for the same pixels as the ones selected for Figs. 5 and 6. A bimodal distribution is visible like Rimmele (2004) in his Fig. 7 but not with the same shape. The gray diamonds represent the high-flux points above 500 Gauss and the small black dots represent the low-flux points with $\left|B_{/ /}\right|<200$ Gauss. For that last one the distribution is more compact around 1.0 in intensity than found by Rimmele (2004) without increasing intensity for the upflowing motions. For the high-flux points above 500 Gauss, we measure a mean velocity two times greater than found by Rimmele (2004) and also we observe important downflows for the brightest one.

The difference between our plots and those of Rimmele (2004) can be due to the difference of spatial resolution ( $0{ }^{\prime} .35$ for us, 0.2 for Rimmele) and to the difference, in small part, of the location of his observation $(\mu=0.998)$ close to the disk center (our observation is done at $\mu=0.91$ ). The line of sight velocities and intensities, of such small features, are affected in particular for the small values of the magnetic field.

\subsection{Time evolution of filigree points}

The quality of our time sequence allows us to follow the properties of the network bright features during their life. Figures 9 and 10 show the time evolution of the intensity (continuum 


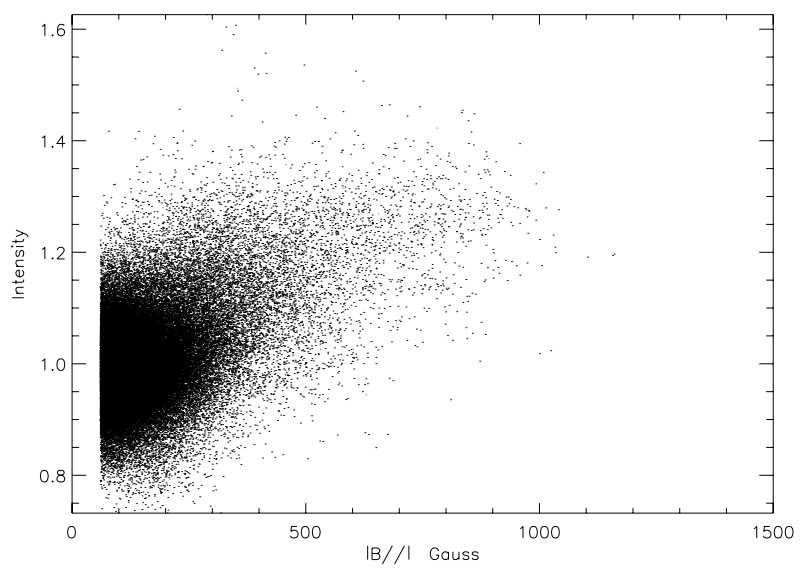

Fig. 6. Intensity at $144 \mathrm{~m} \AA$ versus magnetic field (absolute value) at $288 \mathrm{~m} \AA(170 \mathrm{~km})$ for $\left|B_{/ /}\right|>60$ Gauss.

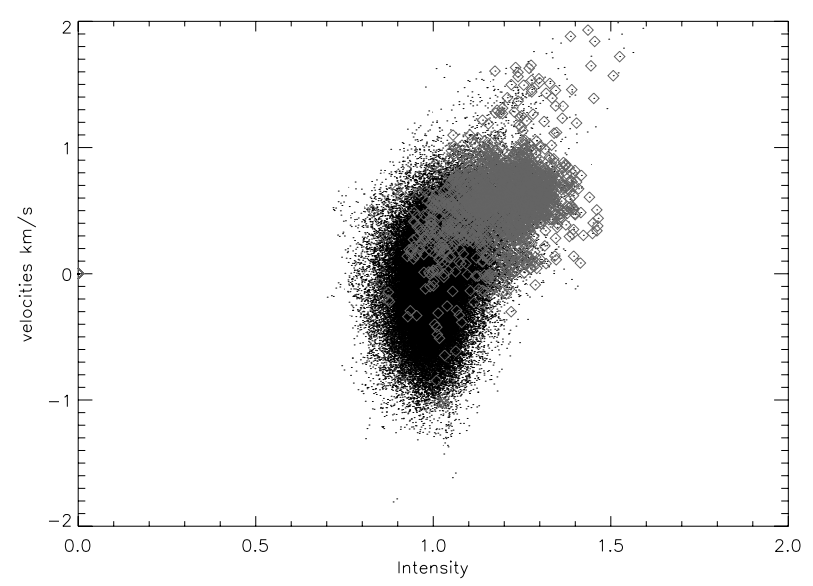

Fig. 7. Velocity at $288 \mathrm{~m} \AA(170 \mathrm{~km})$ versus intensity at $144 \mathrm{~m} \AA$ for $\left|B_{/ \mid}\right|<200$ Gauss (dots) and $\left|B_{/ /}\right|>500$ Gauss (diamonds). Positive velocity denotes downflow.

and line core), magnetic field and Doppler velocity (at 288 and $144 \mathrm{~mA}$ ) of two bright features. These flux tubes are detected by using the $144 \mathrm{~m} \AA$ intensity fluctuations (denoted as "core" intensity).

In the first flux tube A (coordinates 463, 18), we note a quite constant intensity both in the continuum and in the line core during all its life. During all the sequence the magnetic field is always found positive and constant around 700 Gauss and the Doppler velocity indicates a clear downflow location with velocity $-350 \mathrm{~m} / \mathrm{s}$ at $144 \mathrm{~m} \AA$ and $-700 \mathrm{~m} / \mathrm{s}$ at $288 \mathrm{~m} \AA$. The observed velocity fluctuations are well correlated at different depth but do not seem to correspond to magnetic field fluctuations (seeing effects).

The second flux tube B (coordinates 784, 43), is also quite constant in intensity both in the continuum and in the line core during all its life. This flux tube has always a negative magnetic field around -450 Gauss. At $t=16 \mathrm{mn}$ and $t=41$ the different behaviours of the magnetic field at depth $288 \mathrm{~m} \AA$ (dot line) are not significant. The Doppler velocity are also found in majority in the downflow location with velocity between $0 \mathrm{~m} / \mathrm{s}$ and $-350 \mathrm{~m} / \mathrm{s}$. Solar small fluctuations in time are probably due to seeing effects which moves the flux tubes location (+ or -2 pixels).

There are only few measurements available for the magnetic field time evolution in the bright feature. Indeed, this requires extremely high spatial resolution and high cadence of magnetograms. De Pontieu (2002) describes small-scale emerging flux in the photosphere showing an increasing magnetic flux during the emergence phase (around $300 \mathrm{~s}$ ) and a clear decrease in the adjacent dispersion phase (around $420 \mathrm{~s}$ ). The very recent work of Rouppe van der Voort et al. (2005, in their Fig. 5), shows a more or less stable magnetic field in flux tube during at least around $15 \mathrm{~min}$. The apparent magnetic field intensity fluctuations in their figure seems to be generated by the seeing variations between the two instants of the polarimetric measurements.

The measurement of the 20 bright features present in our field of view gives the mean properties:

$$
\begin{aligned}
& V 144=-424 \mathrm{~m} / \mathrm{s} \text { and }|B 144|=416 \text { Gauss }(h=327 \mathrm{~km}) \\
& V 288=-573 \mathrm{~m} / \mathrm{s} \text { and }|B 288|=421 \text { Gauss }(h=170 \mathrm{~km}) .
\end{aligned}
$$

In the quiet area (see right of Fig. 8), the magnetic field does not vary significantly with height (a few \% only), but in bright features it has the tendency to increase gently with altitude, except for the Moving Magnetic Feature (MMF) present near the sunspot. In any case, the gradient is small in bright features and never exceeds 10 to $15 \%$ of the magnitude of the magnetic field. In the spot, the situation is quite different, because the magnetic field decreases strongly with height, -200 Gauss in comparison to a magnitude of 800 Gauss, thus $25 \%$ or more, corresponding to a gradient of the order of $1 \mathrm{G} / \mathrm{km}$. This Fig. 8 can be related to Figs. 1 and 4 of Eibe et al. (2002) and the results seem to be in good agreement.

From a theoretical point of view, models predict an expansion of magnetic flux tubes over height range of $300 \mathrm{~km}$ with a decrease of the magnetic field amplitude (Solanki et al. 1999). This decrease is generally computed in models from various authors (Bercik et al. 2002; Sheylag et al. 2004; Vögler et al. 2005) and gives a decrease lying between 200 and 300 Gauss depending of the model between the height of 327 and $170 \mathrm{~km}$, corresponding to the heights of our observation. In our previous work (Malherbe et al. 2004), the mean intensity of the magnetic field measured in magnetic concentrations at $288 \mathrm{~m} \AA(170 \mathrm{~km})$ and $144 \mathrm{~mA}(327 \mathrm{~km}$ ) were 945 and 480 Gauss (absolute value) respectively, which was a decrease with the altitude compatible (or slightly greater) with model predictions. In our data, we observe a clear depth effect on Doppler velocity but not in the magnetic amplitude between the same altitudes at 327 and $170 \mathrm{~km}$. The difference found between these observations cannot be explained by the location of the target on the sun which are quite identical in term of viewing angle, or by the sunspot ages ( 5 days for Malherbe et al. 2004, one and between 5 to 15 days for the present one). The magnetic structures width could be smaller in the wings of the line than in line core, as found by Mein et al. (2006). We have noticed that at different locations the size of magnetic features at $288 \mathrm{~m} \AA$ is smaller or equal to the size of magnetic features at $144 \mathrm{~m} \AA$. For a given image quality (seeing) this could imply a smaller filling factor at the altitude of formation of the line wings than in the line core which could lead to under evaluate the magnetic field. Then, this could be an explanation of the invariance of the magnetic field with altitude that we oberve. Another possibility is that we observe intrinsic properties of flux tube in which magnetic field decrease more slowly in altitude than expected in active region. In addition, Rimmele (2004) indicates also a slower expansion rate with altitude than predicted by Solanki et al. (1999). 


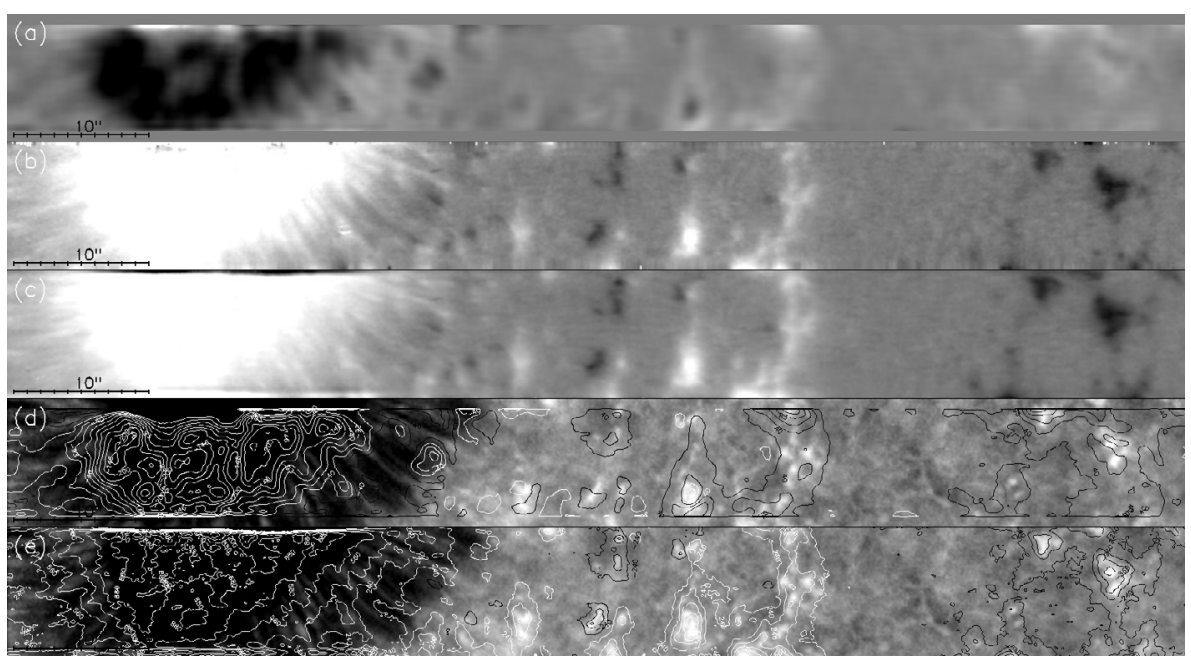

Fig. 8. Observation at time $t=14 \mathrm{mn}$, field of view of $87^{\prime \prime} \times 9^{\prime \prime}$. From top to bottom: a) gradient of the absolute magnetic field between 144 and $288 \mathrm{~m} \AA$. Positive gradient (increasing field with height) in white, negative in dark; b) magnetic field at $288 \mathrm{~m} \AA$ (170 km), after destretching and summing 20 pairs of images $I+V, I-V$. North polarities in white, South in dark; c) magnetic field at $144 \mathrm{~m} \AA$ ( $327 \mathrm{~km})$, after destretching and summing 20 pairs of images $I+V, I-V$; d) intensity at $144 \mathrm{~m} \AA$ showing bright features and filigree, with the gradient of the absolute value of the magnetic field (this is the difference between the absolute value of the magnetic field at 144 and $288 \mathrm{~m} \AA$, after reduction of the size of the pixel to $1^{\prime \prime}$ in order to reduce the noise). Isocontours for \pm 13 Gauss, \pm 40 Gauss, \pm 66 Gauss, \pm 94 Gauss, \pm 120 Gauss, \pm 146 Gauss, \pm 173 Gauss, \pm 200 Gauss, \pm 227 Gauss, \pm 253 Gauss) with positive gradient (magnetic field increasing with height in absolute value) for black and negative gradient (magnetic field decreasing with height) for white; e) intensity at $144 \mathrm{~m} \AA$ showing bright features and filigree, with isocontours of the the magnetic field (isocontours for \pm 80 Gauss, \pm 240 Gauss, \pm 400 Gauss, \pm 560 Gauss, \pm 720 Gauss, \pm 880 Gauss, \pm 1040 Gauss, \pm 1200 Gauss, \pm 1360 Gauss, \pm 1520 Gauss).
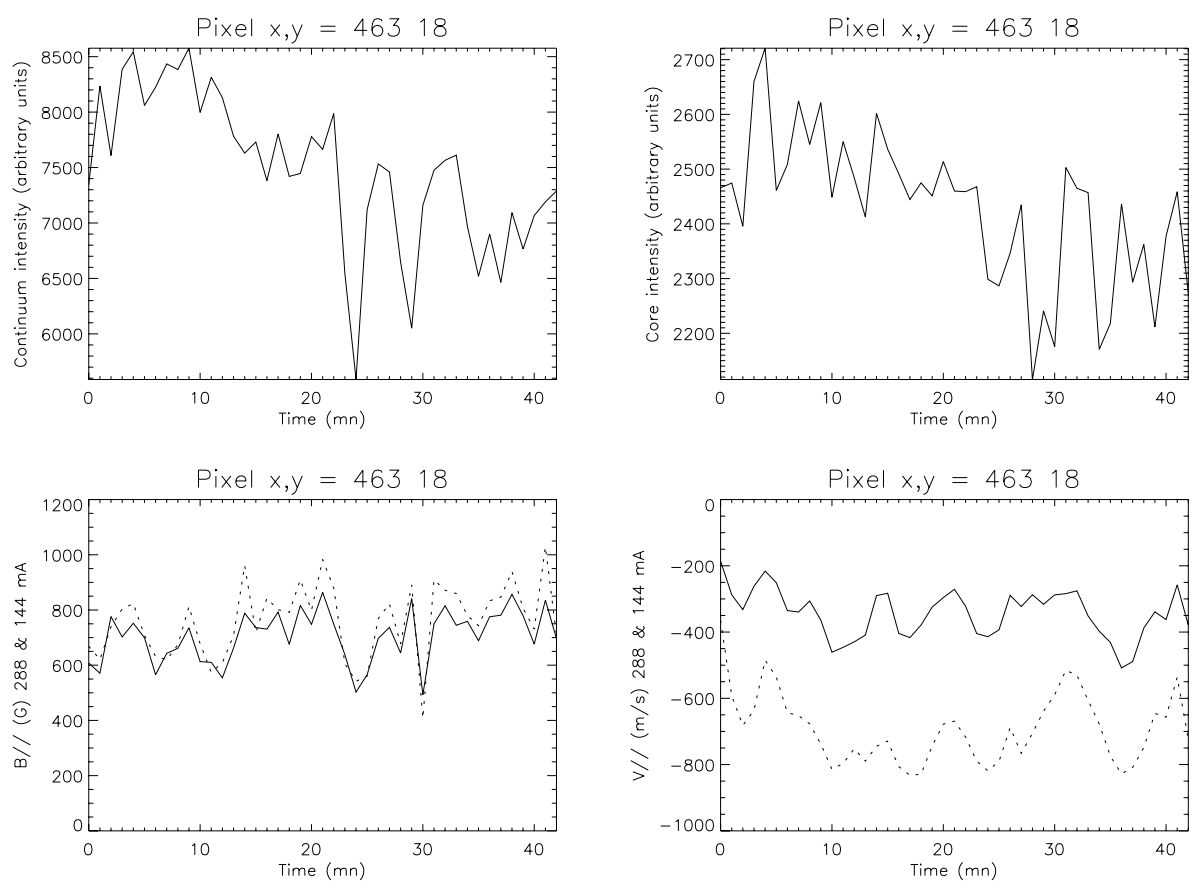

Fig. 9. Magnetic bright feature (A) time evolution, coordinates $(463,18)$ where $V_{/ /}<$ 0 (downward) and $B_{/ /}>0 . B_{/ /}$in Gauss and $V_{/ /}$in $\mathrm{m} / \mathrm{s}$. In the bottom plots, dot and solid lines represent depth at 288 and $144 \mathrm{~m} \AA$ respectively.

\subsection{Observation and simulation comparison}

The simulations produce magnetic fields whose structures are very similar to magnetic Sun (Stein et al. 1999; Cattaneo 1999; Emonet \& Cattaneo 2001; Stein \& Nordlund 2002; Stein et al. 2002; Bercik et al. 2002; Stein \& Nordlund 2003; Stein et al. 2003; Sheylag et al. 2004; Komenko et al. 2005; Vögler et al. 2005). In particular, the numerically simulated magnetograms are in good agreement with those observed on the sun (Sanchez Almeida et al. 2003). Models strongly suggest that non-stationary velocities to be present in mature flux tubes although strong downflows can exist during the field concentration phase (see Rimmele 2004). However, models also predict, thin sheets of downflowing plasma at the egde of the flux tube. Such events have been recently observed with high spatial resolution observations by Rimmele (2004) and Rouppe van der Voort et al. (2005).

More precisely, the latter authors found that the plasma in magnetic elements is basically at rest and sometimes, upflows at $\approx 150 \mathrm{~m} / \mathrm{s}$ within localized concentrations of small magnetic features. The stronger downflows are observed, like Rimmele, in narrow sheets at the edges of the flux sheets. 

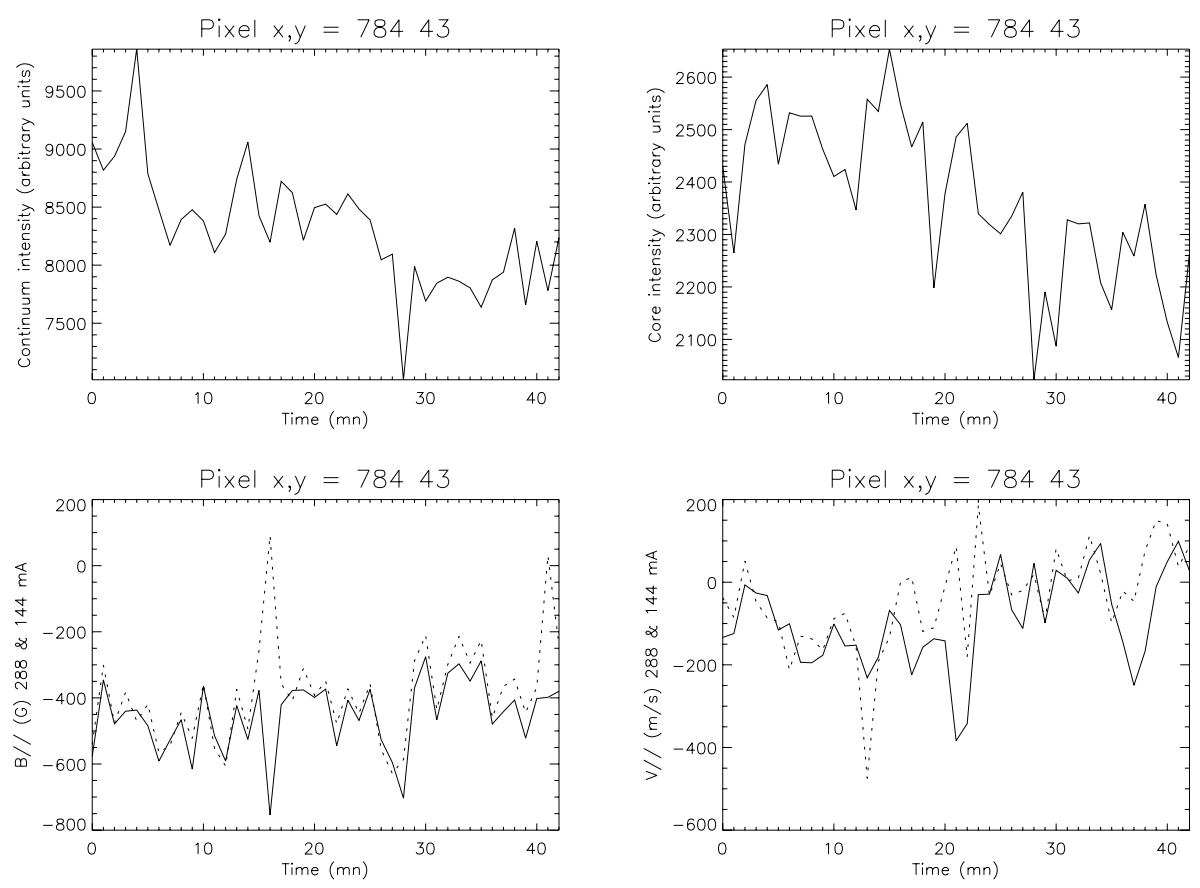

Fig. 10. Magnetic bright feature (B) time evolution, coordinates $(784,43)$ where $V_{/ /}<$ 0 (downward) and $B_{/ /}<0 . B_{/ /}$in Gauss and $V_{/ /}$in $\mathrm{m} / \mathrm{s}$. In the bottom plots, dot and solid lines represent depth at 288 and $144 \mathrm{~m} \AA$ respectively.

In our observations, we do not observe the small upflows or plasma at rest in the flux tube which is probably due to our spatial resolution around 0.35 . The diffraction limit of the telescope leads to mixing of information, as explained by Rimmele (2004), within the flux tube and its surrounding where the downflows are strong. From our observation, we find that each magnetic feature is associated with a strong and concentrated downflow which is in good agreement with the simulation of Stein et al. (2002). The bright feature evolution shows a fairly constant magnetic field along the sequence duration, while downflows may be stable or vary (Figs. 9 and 10).

Regarding now the time evolution of the magnetic field, Stein et al. (2003) show that the surface magnetic field saturate about one turnover time for their computational domain (around $60 \mathrm{mn}$ ) although local dynamo action must exihibit an amplification phase followed by a diffusion process to reconnect and alter the magnetic field and by a recirculation to continue the process. Our measurement during $41 \mathrm{mn}$, of mature flux tubes, does not allow to give any more information about the alteration of these flux tubes by the local convection. The magnetic field in the measured flux tubes is always found fairly constant during all the sequence, this is probably due to our observation of mature flux tubes. The shortness of our time sequence does not allow us to describe all the time evolution of flux tube, longer sequences are necessary for that goal.

\subsection{A particular moving magnetic feature (MMF)}

Let us examine the case of a particular solar magnetic feature during our time sequence Fig. 12. A moving magnetic feature (MMF) is well visible in the penumbra of the spot, with opposite magnetic polarity. During the $41 \mathrm{mn}$ of the sequence, the intensity of the MMF at $144 \mathrm{~m} \AA$ remains almost constant, so that this feature is easy to detect and horizontal motions can be determined precisely. The horizontal drift velocity can be considered as a constant, Fig. 11 , of $0.8 \mathrm{~km} \mathrm{~s}^{-1}$. With such horizontal velocity, this MMF corresponds to the faster class of the MMF described by Zhang et al. (2003) with velocity greater than superganular outflow $\left(0.5 \mathrm{~km} \mathrm{~s}^{-1}\right)$. On the contrary, the line-ofsight velocity derived from dopplershifts (see figure) is not constant and lies in the range -0.6 to $-1.2 \mathrm{~km} \mathrm{~s}^{-1}$ remaining always downward motions. The magnetic field does not exhibit significant variations around a mean value of -300 Gauss (South Polarity, sunspot of North Polarity). The noise in quiet regions was estimated at about 50-60 Gauss, here the noise is higher because it is very hard to follow exactly the same pixel with a time resolution of $1 \mathrm{mn}$ due to seeing fluctuations.

\section{Conclusion}

The detection and the measurement of the magnetic field in the flux tubes is one challenge of the nowadays Solar physics. Several authors (Koschinsky et al. 2001; Domìnguez Cerdeña et al. 2003; Rimmele 2004; Berger et al. 2004; Stangl \& Hirzberger 2005; Rouppe van der Voort et al. 2005) have recently performed magnetic field measurements at very high spatial resolution. However, due to the difficulty to get time sequence with constant high quality, few of them had the possibility to observe the temporal evolution of magnetic flux tubes which are highly linked to the turbulent-convective plasma. The quality of our time sequence allows us to follow the properties of the magnetic concentrations during their life, in intensity (continuum and line core), magnetic field and Doppler velocity at two altitudes $327 \mathrm{~km}$ and $170 \mathrm{~km}$ in the photosphere. A detailed examination of all the bright features visible (in the NaD1 line) in our field of view reveals that all these features are always associated with downflow motions. In our observations, we do not observe the small upflows or plasma at rest in the flux tube and the stronger downflows, like Rimmele (2004), in narrow sheets at the edges of the flux sheets, which is probably due to our spatial resolution around 0.35 . From our observation, we derived the mean properties of the flux tubes during their life. During all the sequence the magnetic field is found more or less constant like the intensity both in the continuum and in the line core. The observed velocity fluctuations are well correlated at different depth but do not seem to correspond to magnetic field 

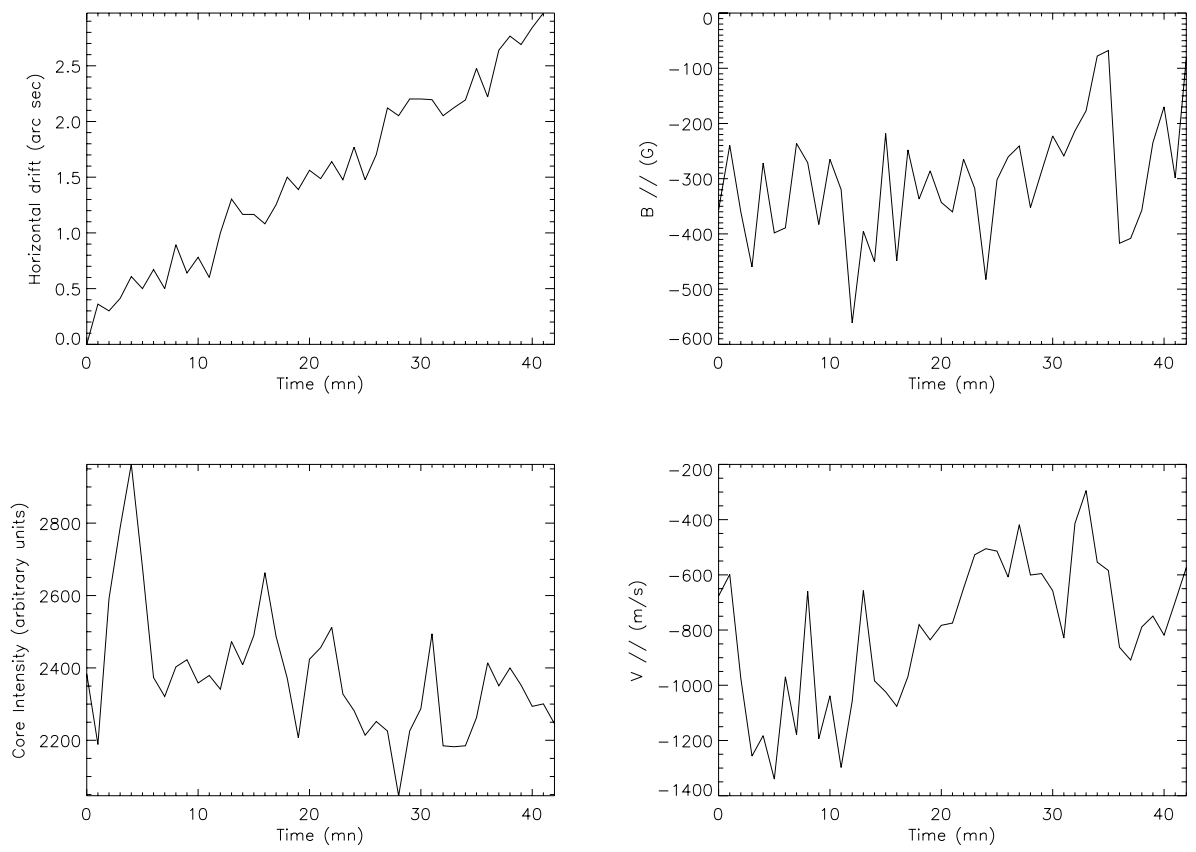

Fig. 11. Time evolution of different parameters of the Moving Magnetic Feature (MMF): $B_{/ /}$ in Gauss and $V_{/ /}$in $\mathrm{m} / \mathrm{s}$. Top left: MMF displacement in arcsec during the sequence; top right: MMF magnetic field at $144 \mathrm{~m} \AA$ time evolution which is fairly constant during the sequence; bottom left: core intensity time evolution of the MMF which is is constant during the sequence; bottom right: MMF Doppler velocity at $144 \mathrm{~m} \AA$, time evolution. We note strong modification of the downflow amplitude between $-0.6 \mathrm{~km} \mathrm{~s}^{-1}$ to $-1.2 \mathrm{~km} \mathrm{~s}^{-1}$ during the sequence.

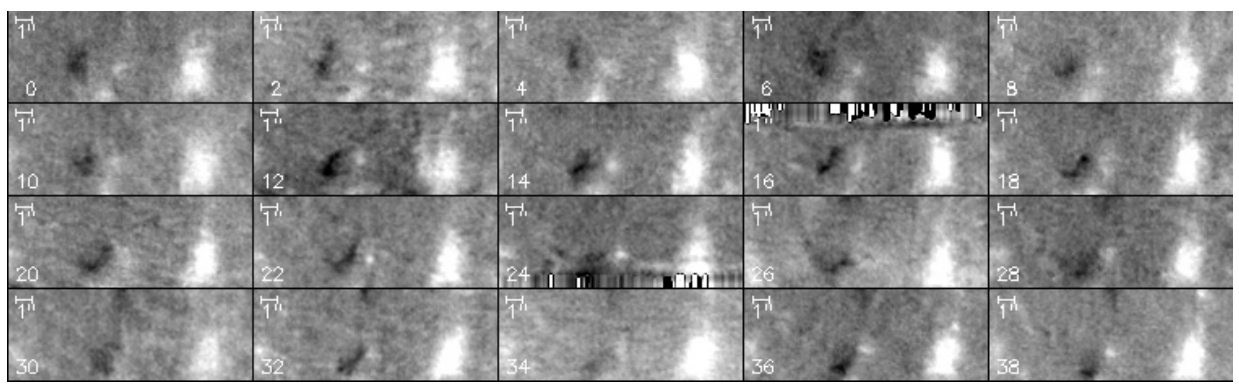

Fig. 12. Moving Magnetic Feature (MMF), dark feature, time evolution (magnetic field). Field of view is $15^{\prime \prime} \times 5^{\prime \prime} .5$, time step is $2 \mathrm{mn}$. North polarities in white, South in dark.

fluctuations (seeing effects). This low fluctuation of the magnetic intensity is probably due to our observation of mature flux tubes.

From measurement of 20 bright features present in our field of view, we derived the mean properties at the height $327 \mathrm{~km}$ of the velocity field $\langle V\rangle=-424 \mathrm{~m} / \mathrm{s}$ and absolute magnetic field value $B=416$ Gauss, and at $170 \mathrm{~km}\langle V\rangle=-573 \mathrm{~m} / \mathrm{s}$ and $B=421$ Gauss. In our previous work (Malherbe et al. 2004), the mean intensities of the magnetic field measured in magnetic concentrations at $170 \mathrm{~km}$ and $327 \mathrm{~km}$ were 945 and 480 Gauss respectively, which was a decrease with altitude compatible (or slightly greater) with model prediction (Solanki et al. 1999). In our present analysis, we observe a clear depth effect on Doppler velocity but not in the magnetic amplitude between the same altitudes at 327 and $170 \mathrm{~km}$. The difference found between these observations is probably related to the difference of filling factor of the magnetic structure at the two considered altitudes or due to the intrinsic properties of flux tubes in different active regions.

We are aware of the small statistic of bright features and the limitation of the conclusion concerning flux tubes evolution properties deduced from this study and Malherbe et al. (2004). Both observations have been performed in quiet sun close to active region. The next step now is to measure the time evolution of the magnetic concentrations in the quiet sun at the disk centre of the Sun, from the birth to the death of concentrated magnetic features, over a large field of view to get a significant statistics. We hope to achieve this goal in 2007 with the improvement of the guiding system of our telescope.

New temporal sequences are necessary at higher spatial resolution and with better spectral resolution in order to improve the determination of magnetic field gradients. New multislit beam shifters with $50 \mathrm{~m} \AA$ of spectral resolution will be available in the future in our spectrograph to acheive this goal.

\section{Additional information}

Images and movies can be found at: http://helios.obspm.fr/malherbe/papers/04JUL15/ index.html

More information about experiment setup (polarimeter, spectrograph, detector) are given by http://helios.obspm. fr/malherbe/papers/index.html

Acknowledgements. This work was supported by the Centre National de la Recherche Scientifique (CNRS, UMR 5572 and FRE 2461) and the Programme National Soleil Terre (PNST). Special thanks are due to the Pic du Midi Observatory staff for their technical assistance. We thank M. Rieutord for useful advice and comments. We wish also to thank the anonymous referee for helpfull comments and suggestions that improved the manuscript. 


\section{References}

Bercik, D. J., Nordlund, Å., \& Stein, R. F. 2002, in Proceedings of SOHO 12 / GONG+ 2002. Local and global helioseismology: the present and future, 27 October-1 November 2002, Big Bear Lake, CA, USA, ed. H. Sawaya-Lacoste, ESA SP-517 (Noordwijk, Netherlands: ESA Publications Division), 201

Berger, T. E., Rouppe van der Voort, L. H. M., Löfdahl, M. G., et al. 2004, A\&A, 428,613

Cattaneo, F. 1999, ApJ, 515, 39

De Pontieu, B. 2002, ApJ, 569, 474

Domìnguez Cerdeña, I., Kneer, F., \& Sànchez Almeida, J. 2003, ApJ, 582, L55

Eibe, T., Aulanier, G., Faurobert, M., Mein, P., \& Malherbe, J.-M. 2002, A\&A, 381,290

Emonet, Th., \& Cattaneo, F. 2001, ApJ, 560, L197

Komenko, E. V., Sheylag, S., Solanki, S. K., \& Vögler, A. 2005, A\&A, 442, 1059

Koschinsky, M., Kneer, F., \& Hirzberger, J. 2001, A\&A, 365, 588

Malherbe, J.-M., Roudier, Th., Mein, P., Moity, J., \& Muller, R. 2004, A\&A, 427, 745

Mein, P. 1981, Proceedings of the japan france seminar on solar physics (Henoux and Moriyama Editors), 285

Mein, P. 2002, A\&A, 381, 271

Mein, P., et al. 2006, A\&A, submitted

November, L. 1986, Appl. Opt. 25(3), 392

Rimmele, T. R. 2004, ApJ, 604, 906
Roudier, Th., Eibe, M. T., Malherbe, J. M., et al. 2001, A\&A, 368, 652

Rouppe van der Voort, L. H. M., Hansteen, V. H., Carlsson, M., et al. 2005, A\&A, 435, 327

Sanchez Almeida, J., Emonet, T., \& Cataneo, F. 2003, ApJ, 585, 536

Sheylag, S., Schussler, M., Solanki, S. K., Berdyugina, S. V., \& Vögler, A. 2004, A\&A, 427, 335

Solanki, S. K., Finsterle, W., Rüedi, I., \& Livingston, W. 1999, A\&A, 347, 27

Stangl, S., \& Hirzberger, J. 2005, A\&A, 432, 319

Stein, R. F., Georgobiani, D., Bercik, D. J., Brandenburg, A., \& Nordlund, Å. 1999, Stellar Structure: Theory and Test of Connective Energy Transport, ASP Conference Series, 173, ed. A. Gimenez, E. F. Guinan, \& B. Montesinos (San Francisco: Astronomical Society of the Pacific), 193

Stein, R. F., \& Nordlund, A. 2002, Solar Surface Magneto-Convection and Dynamo Action, in Magnetic Coupling of the Solar Atmosphere, IAU Coll., 188, ESA Publ. SP-505, 83

Stein, R. F., Bercik, D., Nordlund, Å., \& Aring. 2002, Solar Convection and Magneto-Convection Simulations, Il Nuovo Cimento C, Geophys. Space Phys., 25, 513

Stein, R. F., \& Nordlund, Å. 2003, Solar Surface Magneto-Convection, in Modeling Stellar Atmospheres, ed. N. Piskunov, W. W. Weiss, \& D. F. Gray, ASP IAU Symp., 210, 169

Stein, R. F., Bercik, D., \& Nordlund, Å. 2003, in Current Theoretical Models and High Resolution Solar Observations: Preparing for ATST, ed. A. A. Pevtsov, \& H. Uitenbroek, ASP Conf. Ser., 286, 121

Vögler, A., Sheylag, S., Schüssler, M., et al. 2005, A\&A, 429, 335

Zhang, Jun, Solanki, S. K., Wang, \& Jingxiu 2003, A\&A, 399, 755 